\title{
Der Sänger im Regen
}

\author{
Ein soziologisches Streiflicht \\ auf die Relevanztheorie von Alfred Schütz ${ }^{1}$
}

\section{Andreas Göttlich}

Die Szene gehört zur Standard-Ikonik westlicher Cineastik: Ein Mann verabschiedet sich in einem Hauseingang mit einem Kuss von seiner Geliebten und tritt anschließend mit aufgespanntem Regenschirm hinaus auf das regennasse Trottoir. Trotz eines hernieder gehenden Wolkenbruchs winkt er dem Fahrer des für ihn bereitstehenden Autos ab und beginnt die Straße hinunterzugehen. Nach einigen Schritten, während derer er eine einfache Melodie summend den herabprasselnden Regen beobachtet, schließt er seinen Regenschirm und setzt seinen Weg schlendernd und singend fort. Ungeachtet seines nunmehr unzureichenden Wetterschutzes scheint er - ganz im Gegensatz zu den übrigen Passanten - den heftigen Niederschlag zu genießen. Er beginnt ausgelassen zu tanzen, bis er von ebenso verständnislosen wie argwöhnischen Blicken eines Straßenpolizisten zur Raison gebracht wird, und er verschenkt schließlich seinen für ihn offenbar nutzlosen Regenschirm an einen zufällig daherkommenden Mann.

Bei dem Tänzer handelt es sich selbstverständlich um Gene Kelly, in seinem Rollen-alter-ego Don Lockwood; die Szene entstammt dem Hollywood-Musical „Singin' in the Rain“ aus dem Jahre 1952. So sehr das Verhalten von Don Lockwood innerhalb der fiktionalen Sinnwelt des Musicals als nachvollziehbar erscheint, so ungewöhnlich mutet es doch an, wenn man es an den Routinen alltäglichen Handelns misst. Sucht man nach einer handlungstheoretischen Erklärung für die außergewöhnliche Verhaltensweise, so ist die Motivlage des Protagonisten zu fokussieren, die ihn zu seinem Handeln veranlasst. Gemäß der Relevanztheorie von Alfred Schütz, die im Fokus des vorliegenden Aufsatzes steht, muss dabei zwischen Um-zu- und Weil-Motiv unterschieden werden. ${ }^{2}$ Im Hinblick auf das Um-zu-Motiv Lockwoods könnte man zunächst vermuten, dieser schließe seinen

\footnotetext{
1 Danken möchte ich Thorsten Berndt und Jochen Dreher für kritische Fragen und wertvolle Anregungen.

2 Näheres zu dieser Unterscheidung in Abschnitt I.
} 
Regenschirm, um besser tanzen zu können, doch verträgt sich eine solche Annahme erstens schlecht mit der Tatsache, dass er eine ganze Weile ohne erkennbare Probleme mit aufgespanntem Regenschirm tanzt, und zweitens steht sie im Widerspruch zu dem Umstand, dass Lockwood nach vollendetem Tanz seinen Regenschirm nicht wieder aufspannt, sondern diesen im Gegenteil verschenkt. Offenbar schließt Lockwood seinen Regenschirm demnach gezielt, um nass zu werden. Folgt man Schütz' Theorie, so muss man den Grund für dieses eigentümliche Um-zu-Motiv - immerhin handelt es sich nicht um einen erfrischenden Sommerregen, dem man sich gemeinhin gerne aussetzen würde - in einem konstituierenden Weil-Motiv suchen. Mit Blick auf den Beginn der Szene wird man diesbezüglich schnell fündig: Lockwood ist glücklich verliebt. In diesem Zustand sieht er die Welt durch die sprichwörtliche „rosarote Brille“ und er deutet selbst unerfreuliche Dinge des Lebens (wie den auf ihn herabprasselnden Regen) in Reflexe seiner positiven Grundstimmung um. Eine Strophe des von ihm gesungenen Liedes bringt dies zum Ausdruck:

„I'm laughing at clouds

So dark up above

The sun's in my heart

And I'm ready for love“.

Es ist sicherlich nicht mehr als bloße Koinzidenz, dass Schütz in seinem Buch „Der Sinnhafte Aufbau der sozialen Welt" gerade das Beispiel des Aufspannens eines Regenschirms zur Erläuterung seines Konzepts der Motivationsrelevanzen benutzt (vgl. Schütz 2004a: 203 ff.), ${ }^{3}$ also desjenigen Theorems, das wir soeben auf der Suche nach Don Lockwoods Motivlage bereits angewendet haben. Schütz' Beispiel ist eher dem Alltag entnommen. Er untersucht die Motive, die in der Aussage „Weil es regnet, spanne ich meinen Schirm auf“ (ebd.: 203) enthalten sind. Er stellt fest, dass sich als Weil-Motiv der fraglichen Aktion die Erfahrung des handelnden Subjekts identifizieren lässt, wonach Regen nasse Kleidung verursacht

3 Schütz' Werk erschien ungefähr 20 Jahre vor dem Musical, und man darf umgekehrt wohl ausschließen, dass Betty Comden und Adolph Green (die Drehbuchautoren von Singin' in the Rain) den "Sinnhaften Aufbau“ gelesen haben. Gleichwohl dürfte es sich insofern um keinen Zufall handeln, als bereits Max Weber, auf dessen Werk Schütz aufbaut, in seinen Soziologischen Grundbegriffen mit dem Beispiel des Regenschirm-Aufspannens operiert (vgl. Weber 1980: 11). Da Weber das Beispiel indes nicht auf seine Relevanzstruktur hin analysiert, sondern es vielmehr verwendet, um den Unterschied zwischen sozialem und nicht-sozialem Handeln zu erläutern, kann seine Erörterung hier übergangen werden. 
und diese wiederum ein Gefühl der Unlust hervorruft. Angesichts dieser Erfahrung konstituiert sich im Bewusstsein des Handelnden das Um-zu-Motiv, nicht nass werden zu wollen. Unterstellt man weiter, dass der Handelnde im Sinne eines Rezeptwissens davon Kenntnis besitzt, dass man sich mittels Regenschirmen vor dem Nasswerden schützen kann, folgt aus der beschriebenen Motivverknüpfung das Aufspannen des Regenschirms.

Angesichts des diametralen Gegensatzes zwischen dem Verhalten Don Lockwoods und des Schütz'schen "homunculus“ bietet sich ein Vergleich der beiden Exempel an. Bei ersterem haben wir die folgende Verkettung festgestellt: Verliebtheit als Weil-Motiv, Nass-werden-Wollen als Um-zu-Motiv, Schließen des Regenschirms als resultierende Handlung. Das Äquivalent im Falle des letzteren lautet: Erfahrung des Unlustgefühls bei nasser Kleidung als Weil-Motiv, Nichtnass-werden-Wollen als Um-zu-Motiv, Aufspannen des Regenschirms als resultierende Handlung. So weit, sprich bei paralleler Behandlung der beiden Beispiele, bewährt sich die Schütz'sche Theorie der Motivationsrelevanz im Sinne einer formalen Beschreibungssprache, denn sie erlaubt es, unterschiedlich gelagerte Fälle unter Verwendung derselben Terminologie zu konzeptionalisieren. Hingegen tauchen Fragen und Probleme auf, sobald man die Fälle kontrastiv gegeneinander hält. Geht man nämlich von der naheliegenden Unterstellung aus, dass Don Lockwood die von Schütz beschriebene Erfahrung des Unwohlseins in nasser Kleidung selbst schon einmal gemacht hat und daher ein entsprechendes Weil-Motiv besitzt, so stellt sich unweigerlich die Frage, weshalb sich Lockwood nicht diesem Motiv entsprechend verhält. Hierauf zu antworten, Lockwoods Verliebtheit wiege eben schwerer als jene Erfahrung des Unwohlseins, ist ebenso korrekt wie unbefriedigend, denn sie lässt die entscheidenden Probleme im Dunkeln: Wie genau spielt sich eine derartige Konkurrenz von Weil-Motiven ab? Schlägt sich nur das eine der beiden in einem Um-zu-Motiv nieder, oder folgt aus dem Widerstreit der Weil- ein analoger Konflikt von Um-zu-Motiven? Und welche Rolle spielt der Wille des Handelnden in diesem Streit? Trifft er eine bewusste Wahl oder erfolgt die Handlungsentscheidung quasi-automatisch?

Fragen dieser Art verweisen auf eine Unterscheidung, die Schütz parallel zu derjenigen zwischen Weil- und Um-zu-Motiven einführt. Er definiert jene als „auferlegt“, diese hingegen als „frei“. Im Hintergrund dieser Konzeption steht die Überlegung, dass in der Vergangenheit liegende Motive nicht zur freien Disposition des handelnden Subjekts stehen, in die Zukunft gerichtete Handlungsentwürfe hingegen schon. Thema des vorliegenden Aufsatzes ist eine Diskussion von Schütz' genereller Gegenüberstellung von „auferlegten“ und „freien“ Relevanzen, d.h. nicht nur mit Bezug auf Motivationsrelevanzen, sondern auch hinsichtlich 
der übrigen von Schütz eingeführten Relevanztypen: der sogenannten thematischen sowie der Interpretationsrelevanzen. Hierfür ist es unerlässlich, vorab die Schütz'sche Relevanztheorie in ihrem thematischen Kontext vorzustellen.

\section{Die Relevanztheorie von Alfred Schütz}

Die folgende Darstellung rekurriert zum einen auf das posthum veröffentlichte Relevanzmanuskript von Alfred Schütz selbst (Schütz 2004b), zum anderen auf die Ausführungen eines seiner Schüler, Thomas Luckmann, der die Relevanztheorie in einem gesonderten Kapitel der "Strukturen der Lebenswelt“ (Schütz/Luckmann 2003: $252 \mathrm{ff}$.) behandelt; einer Schrift, die er auf der Grundlage von Entwürfen seines Lehrers verfasste. ${ }^{4}$ Schütz' Relevanztheorie ist Teil seiner Theorie der Lebenswelt, welche den "Gesamtzusammenhang der Lebenssphäre“ (Schütz 1971: 284) ${ }^{5} \mathrm{zu}$ analysieren sucht. Diese Analyse wird aus streng subjektiver Perspektive betrieben, doch führt sie zur Aufdeckung allgemeiner Strukturen, die konkreten Bewusstseinsinhalten situierter Subjekte zu Grunde liegen. Das Konzept der Lebenswelt gerät somit zum allgemein anwendbaren Theorieinstrumentarium. $\mathrm{Zu}$ seinen generellen Charakteristika zählen die Aufschichtungen in räumlicher, zeitlicher und sozialer Dimension (vgl. Schütz/Luckmann 2003: 51 ff.) wie auch die topographische Untergliederung nach Relevanzen. Das Relevanzsystem eines jeden Menschen ist vertikal unterteilt in Zonen höherer und niederer Relevanz sowie horizontal in Bereiche verschiedenartiger Relevanzen.

In diesem Sinne stellt das Relevanzproblem eines der zentralen Themenfelder einer Soziologie dar, die mit Max Weber soziales Handeln verstehen und dadurch ursächlich erklären will: „Alle Erfahrungen und alle Handlungen gründen in Relevanzstrukturen. Jede Entscheidung stellt außerdem den Handelnden mehr oder minder explizit vor eine Reihe von Relevanzen. Das Relevanzproblem ist vielleicht das wichtigste und zugleich schwierigste Problem, das es in der Beschreibung der Lebenswelt zu lösen gibt“ (ebd.: 253). Insofern soziales Handeln also wesentlich durch Relevanzsetzungen beeinflusst wird, sind diese als explanans zu seiner Erklärung heranzuziehen. Doch können Wertzuschreibungen im Sinne von Webers

4 Trotz vereinzelter Unterschiede im Detail können die Texte von Schütz und Luckmann für den vorliegenden Zweck als Einheit behandelt werden - von einer bedeutsamen Ausnahme abgesehen, auf die in Abschnitt III eingegangen wird.

5 Eine neuere Übersetzung des zitierten Textes formuliert: „das ganze Universum des Lebens“ (Schütz 2003b: 224). 
„,innerem Verhalten“ selbst als (soziale) Handlungen konzipiert werden, das bedeutet, sie sind selbst explanandum soziologischer Forschung.

Bekanntlich versteht Schütz seine theoretischen Bemühungen als Fortentwicklung der Verstehenden Soziologie Webers. Er kritisiert an dessen Soziologischen Grundbegriffen die unzureichende philosophische Fundierung und er sucht, diesem Mangel durch die Berücksichtigung phänomenologischer Betrachtungen - v. a. der Bewusstseinsanalysen Edmund Husserls - zu begegnen. Mittels des Rückgangs auf subjektive Bewusstseinsakte beabsichtigt er, zentrale Begrifflichkeiten wie Sinn, Handlung oder eben auch Relevanz ${ }^{6}$ aufzuklären. Die Analyse der sozialen Konstruktion soll durch diejenige der individuellen Konstitution vorbereitet werden. Entsprechend dieser Absicht beschreibt Schütz im Rahmen seiner Relevanztheorie, wie sich im Bewusstsein von Subjekten Wertzuschreibungen konstituieren: Wie erlangt ein Thema Aufmerksamkeit, welche seiner Aspekte werden als bedeutsam erkannt, welche Wissensbestände werden zu seiner Auslegung herangezogen, welche Motive nehmen hierbei Einfluss? Wesentlicher Bestandteil dieser Beschreibung ist die im Vorigen erwähnte Unterscheidung von „freien“ und „erzwungenen“ Relevanzen, d. h. die Frage, inwiefern dem Subjekt die von ihm vollzogenen Wertzuschreibungen vorgegeben sind.

Aus dem Gesagten wird klar, dass Schütz das Relevanzproblem im Zusammenhang von Deutungsprozessen behandelt. Die menschliche Welt, in der wir handeln, ist für ihn immer schon eine gedeutete Welt: „Genau genommen gibt es nirgends so etwas wie reine und einfache Tatsachen. Alle Tatsachen sind immer schon aus einem universellen Zusammenhang durch unsere Bewußtseinsabläufe ausgewählte Tatsachen. Somit sind sie immer interpretierte Tatsachen" (Schütz 2010: 333). Das generelle Ziel von Deutungsprozessen besteht in der Überführung von Fremdheit in Vertrautheit. Ein neues Ereignis wird mit bestehenden Erfahrungsbeständen abgeglichen und unter vertraute Auslegungsschemata subsumiert, wobei sich diese Einordnung an der Realität bewähren muss, soll heißen, die aus ihr folgenden Erwartungen müssen erfüllt werden. So sind neue Ereignisse nicht zwangsläufig auch neuartig, denn man kann mit typischen Charakteristika des Neuen durchaus bereits vertraut sein: „Vertrautheit bezeichnet ... die Möglichkeit, neue Erfahrungen hinsichtlich ihres Typus auf den habituellen Besitz schon erworbenen Wissens zu beziehen" (Schütz 2004b: 118). Der Mensch greift also im Zuge von Deutungsprozessen auf Wissensbestände zurück, die sich

6 Dem Begriff der Relevanz entspricht in Webers Terminologie derjenige des Wertbezugs. Die Behauptung einer diesbezüglichen thematischen Kontinuität zwischen den Werken von Weber und Schütz folgt den Interpretationen von Ilja Srubar (2007) und Elisabeth List (2004). 
in früheren Auslegungsakten gebildet und bewährt haben. Für den letzteren Vorgang wählt Schütz den Terminus „Wissenssedimentierung“.

Schütz interessiert nun, wie sich im Bewusstsein des seine Umwelt auslegenden Individuums ein Thema, ein Wissensbestand oder ein Motiv als relevant konstituiert. Dementsprechend unterscheidet er zwischen drei grundlegenden Typen von Relevanz: (1) thematische Relevanzen, (2) Auslegungs- oder Interpretationsrelevanzen - unterteilt in solche des Themas und solche des Wissensvorrats sowie (3) Motivationsrelevanzen. Bei dieser Differenzierung handelt es sich im Sinne Webers um eine idealtypische, d. h. sie dient dem Zweck der Theoriebildung und beansprucht nicht, die Wirklichkeit unmittelbar abzubilden. Jeder der drei Typen wird nochmals unterteilt in erzwungene und freiwillig gewählte Relevanzen. Erstere heißen bei Schütz „auferlegte“ oder „gebundene“, letztere „motivierte“, „freie“, „intrinsische“ oder auch „wesentliche“ Relevanzen. ${ }^{7}$

(1) Die thematische Relevanz bestimmt, was einem Menschen zum Thema wird, worauf er seine Bewusstseinstätigkeit richtet. Wie eine thematische Relevanz zustande kommt, entscheidet über ihre Einordnung als auferlegt oder wesentlich. Bei auferlegten thematischen Relevanzen handelt es sich prinzipiell um Begebenheiten, die das Feld des Vertrauten verlassen; sie werden im Allgemeinen durch Modifikationen bedingt, welche die lebensweltlichen Idealisierungen des „Undso-weiter" sowie des „Ich-kann-immer-wieder“ (vgl. Schütz/Luckmann 2003: 34) unterbrechen, im Speziellen auch durch soziale Interaktion. Wesentliche thematische Relevanzen bezeichnen die willentliche Einteilung des Bewusstseinsfeldes in einen thematischen Kern und einen horizontartigen Hintergrund und ergeben sich infolge (a) des Wechsels oder (b) der Entfaltung eines bestehenden Themas. In ersterem Falle wird ein Thema neu gesetzt, in letzterem werden Implikationen des inneren und äußeren Horizontes eines Themas fokussiert. Es handelt sich um „Sub-Thematisierungen“ (ebd.: 268), deren Richtung durch die Vorgeschichte des thematischen Feldes im Wesentlichen vorgegeben ist.

(2) Wird eine Sache als Unvertrautes thematisiert, so muss sie ausgelegt werden, wobei die sogenannten Auslegungs- oder Interpretationsrelevanzen zum Tragen kommen. Mit diesem Begriff werden sowohl diejenigen Aspekte des Themas bezeichnet, die für die Auslegung relevant werden, als auch diejenigen Elemente des zuhandenen Wissensvorrats, die bei der Auslegung zur Anwendung kommen. Luckmann nennt dies den „Doppelcharakter“ (ebd.: 274) der Interpretationsrelevanz. Nicht sämtliche Aspekte eines Themas sind für seine aktuelle Auslegung von

7 Die Adjektive variieren sowohl bei Schütz selbst als auch zwischen den Übersetzungen seiner Texte aus dem Englischen. 
Bedeutung, wobei es dem Auslegenden bis zu einem gewissen Grad vorgegeben ist, auf welche thematischen Aspekte er einzugehen hat. Auch werden für die Interpretation nur bestimmte Wissenselemente berücksichtigt. Es sind jene früher gemachten Erfahrungen des Auslegenden relevant, die dem noch unvertrauten Thema in irgendeiner Weise ähneln, die also eine Typik bereitstellen, unter welche das Unvertraute subsumiert werden kann. Neben der aktuellen Situation des Auslegenden kommt daher seinem biographischen Erfahrungsschatz große Bedeutung zu. Geschieht die Auslegung des neuen Themas quasi-automatisch, stellt sich mit anderen Worten eine routinemäßige Deckung mit dem vorhandenen Wissensvorrat ein, so liegt eine auferlegte Interpretationsrelevanz vor. Reichen indes die Übereinstimmungen mit dem vorhandenen Wissen nicht aus, um zu einer adäquaten Deutung zu gelangen, so entsteht ein Auslegungsproblem, welcher Fall als motivierte Interpretationsrelevanz beschrieben wird. Gründe hierfür können sein: (1) Eine aktuelle Erfahrung passt nicht in den vertrauten Typus, (2) die Bestimmtheit des Typus genügt nicht zur Situationsbewältigung, (3) eine aktuelle Erfahrung offenbart die Unverträglichkeit zweier Elemente des bestehenden Wissensvorrats. Der Auslegungsvorgang selbst kann aus verschiedenen Momenten bestehen: (a) der Themenentwicklung, (b) einer Sub-Thematisierung, (c) der Herbeischaffung von auslegungsrelevantem Material sowie (d) dem "Abtasten“ relevanter Elemente des vorhandenen Wissensvorrats. Bis zu welchem Gewissheitsgrad Auslegungsrelevanzen entwickelt werden (müssen), hängt vom aktuellen Interesse $\mathrm{ab}$, das wiederum von der Situation bestimmt wird.

(3) Damit ist bereits auf den dritten Typus, die Motivationsrelevanz verwiesen. Mit Weber definiert Schütz das Motiv allgemein als sinnhaften Grund von Verhalten (vgl. Weber 1980: 5), doch differenziert er über Weber hinausgehend zwischen zwei prinzipiell verschiedenen Formen des Motivs: „Erstens erscheint mir als sinnhafter Grund meines Verhaltens eine Serie von Erwartungen künftigen Geschehens, welches sich in Auswirkung meines Verhaltens vollziehen wird. ... Zweitens aber erscheinen mir als sinnhafter Grund meines Verhaltens diejenigen meiner abgelaufenen Erlebnisse, welche mich dazu geführt haben, eben dieses Verhalten an den Tag zu legen“" (Schütz 2004a: 111; Hervorhebungen im Original). Der Begriff „Motivationsrelevanz“ bezeichnet mithin entweder die Bedeutsamkeit, die im Hinblick auf zukünftiges, im Zuge eines Entwurfs als vollzogen imaginiertes Handeln besteht, in welchem Fall von einem „Um-zu-Motiv“ gesprochen wird. Oder die Relevanzzuschreibung lässt sich aus Dispositionen ableiten, die biographisch, also in der Vergangenheit angelegt sind, was als "Weil-Motiv“ bezeichnet wird. Um-zu- und Weil-Motive sind miteinander verknüpft, insofern in die Zukunft gerichtete Handlungsentwürfe stets auf in der Vergangenheit liegen- 
de Dispositionen verweisen. Weil-Motive werden weiter in „echte“ und „unechte“ unterteilt. „Unechte“ Weil-Motive sind solche, die in Um-zu-Motive umformuliert werden können. Zur entsprechenden, im Alltag häufig beobachtbaren Konfusion von Um-zu- und Weil-Motiven kommt es laut Schütz, wenn aus einer zukünftigen Perspektive auf einen vergangenen Handlungsentwurf geblickt wird (vgl. ebd.: 195 ff.; Schütz/Luckmann 2003: 286 ff.). Die Unterscheidung von Um-zuund Weil-Motiv entspricht schließlich derjenigen zwischen „wesentlichen“ (i.e. „freien“) und „auferlegten“ Motivationsrelevanzen. Luckmann formuliert, „daß innerhalb dieser Struktur ... zwei Formeln zu finden sind, eine, freie und eine ,gebundene. Die erste ist die vom Handlungsentwurf in die Zukunft bestimmte Motivationskette, die zweite die biographische, durch sedimentierte Motive bestimmte ,Einstellung““ (ebd.: 287). „Frei“ ist eine Motivationsrelevanz demnach ausschließlich dann, wenn sie sich auf einen volitiven Entwurf bezieht, und das auch nur im Vollzug dieses Entwurfs. Von einem einmal aufgestellten Entwurf abgeleitete Motivationsrelevanzen werden dagegen als auferlegt konzipiert.

Wiederholt betont Schütz die enge Verflochtenheit der drei lediglich analytisch zu trennenden Relevanztypen. So können Motivationsrelevanzen die anfängliche Situationsbestimmung, sprich die thematischen Relevanzen beeinflussen, und sie lenken darüber hinaus die Aufmerksamkeit des Auslegenden, d.h. dessen Interpretationsrelevanzen. Wichtig ist ferner, dass zwischen den Relevanztypen keinerlei Rang- oder Reihenfolge besteht. Ein Vorher und Nachher existiert ausschließlich im reflexiven Zugriff.

So weit gilt die phänomenologische Beschreibung einzelnen Akten von Relevanzzuschreibungen. Diese verbinden und verfestigen sich zu beständigen Relevanzsystemen, wenn sie sich in pragmatischer Hinsicht bewähren, das bedeutet, wenn sie das notwendige Wissen für die Bewältigung konkreter Problemlagen bereitstellen. Ein Relevanzsystem gibt dem Individuum mithin vor, welche thematischen Aspekte eines Problems es zu beachten und welche Wissensbestände es bei dessen Lösung anzuwenden hat. Schütz spricht in diesem Zusammenhang von Rezeptwissen. Dieses bezieht sich auf solche Probleme, mit denen sich das Individuum immer wieder in vergleichbarer Form konfrontiert sieht und für die es daher Deutungs- und Lösungsroutinen entwickelt.

Der Übergang von der subjektiven zur intersubjektiven Ebene findet dort statt, wo die situationstypischen Probleme zwischen Individuen geteilt werden. Jede Form von Gemeinschaft oder Gesellschaft kennt charakteristische „Krisen“, denen sich ihre Mitglieder immer wieder ausgesetzt sehen. Daher entwickeln Zugehörige gleicher bzw. ähnlicher sozialer Welten oder Milieus zwar nicht identische, aber doch vergleichbare Relevanzsysteme. Luckmann spricht von der „Möglich- 
keit ..., daß sich in sozialen Gruppen und Gesellschaften, die mit typisch ähnlichen Situationen konfrontiert sind und ein typisch ähnliches Schicksal erleiden, typische Betrachtungsstile für Handlungsabläufe und Motivationszusammenhänge ausbilden“ (ebd.: 294). Soziale Gruppen zeichnen sich demnach wesentlich dadurch aus, dass sich die Relevanzsysteme ihrer Mitglieder in typischer Art und Weise ähneln. Derartige Konvergenzen sind essentiell für soziale Kohäsion, ihre Unterstellung ist notwendige Voraussetzung für jegliche Art von Verständigung. ${ }^{8}$ Weiterhin wird das in den gemeinsamen Relevanzsystemen enthaltene Wissen innerhalb der Gruppe tradiert und derart mit zusätzlichem „Gewicht“ ausgestattet. Solch sozial erworbenes Wissen zeichnet sich laut Schütz typischerweise dadurch aus, dass die einzelnen Schritte, innerhalb derer es sich einst konstituierte, in Vergessenheit geraten. Dies geht zu Lasten der Klarheit und Deutlichkeit des Wissens, das stattdessen infolge der Autorität, die ihm aufgrund seiner sozialen Billigung zukommt, akzeptiert wird.

Derartige intersubjektiv geltende, sozial verfestigte Relevanzsysteme sind Gegenstand soziologischer Betrachtung. Sie strukturieren Kulturen, Herrschaftssysteme, Ökonomien etc., weshalb ihre empirische Rekonstruktion zum primären Aufgabenbereich soziologischer Forschung gehört. ${ }^{9}$ Einen wesentlichen Aspekt bilden die in den Relevanzsystemen enthaltenen Momente von Zwang und Freiwilligkeit, speziell im Hinblick auf die Frage der Kontinuität respektive Veränderlichkeit. Das Interesse der Soziologie gilt mithin „objektivierten“ Relevanzsystemen, die von den Mitgliedern einer sozialen Gruppe wechselseitig anerkannt werden, ob aufgrund von Legitimitätsglauben, als Folge von Zwang oder im Sinne latenter Internalisierung. Im Sinne des methodologischen Individualismus ${ }^{10}$ un-

8 Vgl. für den letzteren Punkt Schütz’ „Generalthesis der Reziprozität der Perspektiven“ (2003a: 52 f., 2010: 340 ff.) und hier die „Idealisierung der Kongruenz der Relevanzsysteme“, speziell in der erweiterten Fassung in Schütz' Symbol-Aufsatz (2003a: 163 ff.).

9 Webers Untersuchungen zum Zusammenhang von protestantischer Werthaltung und abendländischem Rationalismus (vgl. Weber 1988) lassen sich als prominentes Beispiel für die soziologische Analyse gesellschaftlicher Relevanzsysteme lesen. In die Terminologie von Schütz übertragen, lautet Webers These, dass die lutherisch-calvinistische Religionsgemeinschaft ein gemeinsames System von Relevanzen teilt. Das bedeutet, dass sie dem gleichen Wirklichkeitsbereich thematische Relevanz beimaß (Berufsleben), bei dessen Interpretation ähnliche Wissensbestände anwandte (Prädestinationslehre) und dabei vergleichbaren Motiven folgte (Heilssuche). Die entsprechende Ethik erwies sich als in besonderem Maße rationalisierungsfähig und brach daher Bahn für den okzidentalen Rationalismus.

10 In der Formulierung Webers: „Handeln im Sinn sinnhaft verständlicher Orientierung des eignen Verhaltens gibt es für uns stets nur als Verhalten von einer oder mehreren einzelnen Personen“ (1980: 6; Hervorhebung im Original). In Schütz' Terminologie entspricht dem das sogenannte „Postulat der subjektiven Interpretation“ (2010: 375). 
tersucht Schütz die Grundlage solcher gesellschaftlichen Konstruktionen in den Relevanzsetzungen von Individuen, d.h. auf der Ebene der subjektiven Konstitution. Von daher erklärt sich seine phänomenologisch orientierte Auseinandersetzung mit dem „Problem der Relevanz“ (Schütz 2004b).

\section{Das Problem}

Nach dieser Referierung der Grundaussagen der Schütz'schen Relevanztheorie kann die Problematik, auf welche die eingangs beschriebene Szene aus „Singin' in the Rain" hinweist, näher betrachtet werden. In Schütz’ Beispiel des Aufspannens des Regenschirms gehen dieser Handlung folgende Bewusstseinssynthesen voran: „Ich nehme wahr, daß es regnet. An dieses Wahrnehmen ,knüpft sich' die Vorstellungsreihe an, daß mit dem Regen ein Naßwerden und dadurch ein Unlust erregender Zustand eintreten könne .... Scheinbar konstituiert sich also in meinem Bewußtsein aus der Wahrnehmung des Regens der Entwurf zu einem Abwehrhandeln" (Schütz 2004a: 204). Dieses in der Vergangenheit verankerte Weil-Motiv ist dem Handelnden laut Schütz „auferlegt“, insofern es biographisch bedingt ist. Es handelt sich um eine Einstellung, die als „motivationsmäßiger habitueller Besitz“" (Schütz/Luckmann 2003: 298) während des Handelns nicht frei disponierbar ist. Mit dem im Regen singenden und tanzenden Don Lockwood präsentiert uns „Singin' in the Rain“ ein Gegenbeispiel, d. h. einen Menschen, der sich offenkundig ohne größere Willensanstrengung über das ihm vermeintlich „auferlegte“ Motiv hinwegsetzt. ${ }^{11}$ Die Szene stellt die relevanztheoretische Verwendung des Adjektivs "auferlegt" mithin in Frage. Gleiches tut der Common Sense, der das Unlustgefühl angesichts nasser Kleidung nicht unbedingt als eine Form von Zwang auffassen würde, dem das Subjekt unterliegt. ${ }^{12}$ Bei der von Schütz vorgenommenen Bestimmung von „auferlegten“ und „freien“ Relevanzen handelt es sich, so kann man

11 Zur Verteidigung der relevanztheoretischen Sprachregelung genügt es nicht, auf den idealtypischen Charakter von Schütz' Unterscheidung hinzuweisen. Don Lockwood wäre demnach kein Gegenbeispiel gegen die Auferlegtheit des Weil-Motivs, sondern einfach eine (durch Zusatzannahmen erklärbare) empirische Ausnahme bzw. Abweichung. Doch geht es hier um die prinzipielle Berechtigung der Identifikation von Weil- mit auferlegten Motiven.

12 An anderer Stelle erwähnt Schütz, der spezifische Komplex von Weil-Motiven eines Individuums könne in psychologischer Wendung als „Charakter“ bezeichnet werden (vgl. Schütz 2004b: 123). In unserem Alltagsverständnis betrachten wir hingegen unseren Charakter nicht unbedingt als etwas, das uns prinzipiell und ausschließlich „auferlegt“ ist. Vielmehr pflegen wir die Vorstellung, unser Charakter befinde sich wenigstens teilweise innerhalb unserer Verfügungsgewalt und sei daher durch unser Zutun formbar. 
formulieren, um eine spezifische Redeweise, die mit unserem alltagssprachlichen Verständnis von Freiheit und Zwang nicht ohne weiteres kurzgeschlossen werden darf. Anschaulich illustriert wird diese Kluft durch die Darstellungsweise in den „Strukturen der Lebenswelt“, wo von „auferlegten“ und „freien“ Motiven stets in Anführungszeichen die Rede ist. Man darf vermuten, dass mit dieser Praxis eben die genannte Differenz indiziert werden soll.

Dieses Auseinanderdriften der Sprachregelungen von Relevanztheorie und Alltag lässt sich weiter ausführen. So erkennt Schütz, wie eingangs bereits beschrieben, im Beispiel des Aufspannens des Regenschirms neben dem Weil- zugleich ein Um-Zu-Motiv als wirksam. Dieses „Um-zu-Motiv kann etwa in dem Satz: ,Um mich vor Nässe zu schützen, spanne ich meinen Schirm auf', ausgedrückt werden“ (Schütz 2004a: 203). Es handelt sich in der Sprachregelung der Relevanztheorie per definitionem um ein „wesentliches“ oder "freies“ Motiv, indem es sich auf den Entwurf eines vom Subjekt für die Zukunft beabsichtigten Handelns bezieht, denn „nur die Wahl des ausgezeichneten Entwurfs ... entspringt aus seiner eigenen volitiven Handlung" (Schütz 2004b: 112). Schütz' Analyse des Regenschirm-Beispiels illustriert mithin, dass aus Sicht der Relevanztheorie einund dieselbe Handlung nicht nur zugleich freiwillig und erzwungen sein kann, sondern sein muss, denn Handelnde besitzen stets sowohl "gebundene"Weil- als auch „wesentliche“ Um-Zu-Motive. Die Einstufung einer Handlung als freiwillig oder erzwungen gerät so zu einer Frage der Betrachtungsweise:

„Kurzum, Pläne sind in Planhierarchien eingebettet, die letztlich auf die Grenzen der menschlichen Situation in der Lebenswelt verweisen. Dies bedeutet, daß entweder unmittelbar oder zumindest mittelbar alles Verhalten in Zusammenhänge der ,freien Motivationsrelevanz eingeordnet werden kann. Umgekehrt hat aber prinzipiell jede Handlung und alles Verhalten eine ,Geschichte. Ein ,erster 'Entwurf ist - solange wir uns mit der Beschreibung der Lebenswelt begnügen - unvorstellbar. Grundsätzlich kann demnach alles Verhalten und jede Handlung in Zusammenhängen der ,gebundenen' Motivationsrelevanz verstanden werden" (Schütz/Luckmann 2003: 304).

Es liegt auf der Hand, dass ein solches Resultat theoretischer Reflexion mit dem Common Sense in Konflikt gerät, der (naiverweise?) in den meisten Fällen von einer eindeutigen Entscheidbarkeit der Frage nach der Freiwilligkeit oder Erzwungenheit einer Handlung ausgeht. Zudem ist diese theoretische Position 
wenig hilfreich im Hinblick auf die empirische Feststellung des Zwangscharakters sozialer Handlungen, an welcher der soziologischen Analyse gelegen ist. ${ }^{13}$

Wenn wir uns vom bisher fokussierten Beispiel des Aufspannens eines Regenschirms lösen, wird der Widerspruch der relevanztheoretischen Terminologie zur alläglichen Sprachregelung noch deutlicher. Man muss nicht lange nachdenken, um Beispiele zu finden, in denen Weil-Motive vom handelnden Subjekt nicht als auferlegt und Um-zu-Motive nicht als freiwillig erfahren werden. Nehmen wir etwa den simplen Fall eines Kaufvertrags über ein beliebiges Objekt. Bei entsprechender Kenntnis wird man sicherlich die in der Biographie des Käufers angelegten Dispositionen rekonstruieren können, die ihn letztlich zum Kauf veranlasst haben, doch wird man deswegen kaum sagen, er sei den Vertrag unfreiwillig eingegangen - ja die Vertragsidee setzt per se Freiwilligkeit voraus. Oder nehmen wir das Beispiel, dass das Opfer in einer Gerichtsverhandlung über den Fall einer Nötigung aussagt, es habe sich nicht gewehrt, um nicht geschlagen oder gar getötet zu werden. Man wird sich schwer tun, hier von einem „freien“ Motiv zu sprechen. Nicht nur dem juristischen Verständnis widerspricht die axiomatische Festlegung von Weil-Motiven als „auferlegt“ und Um-zu-Motiven als „frei“.

Auch im Hinblick auf die Verknüpfung von Weil- und Um-zu-Motiven erscheint die relevanztheoretische Sprachregelung dem Common Sense merkwürdig. Laut Schütz verweist jedes Um-zu- auf ein konstituierendes Weil-Motiv, und das bedeutet in der Konsequenz, dass jedes "wesentliche“ Motiv in einem „gebundenen" Motiv wurzelt. Ist es aber statthaft, einen aus unfreiwilligen Gründen heraus gefassten Handlungsentwurf als "frei“ zu bezeichnen? Diese Merkwürdigkeit zeigt sich nicht zuletzt im Hinblick auf das, was Schütz als „Wirkensbeziehung“ bezeichnet, für welche es wesentlich ist, dass der „auf ein Du zu Handelnde die Um-zu-Motive seines eigenen Handelns als echte Weil-Motive des erwarteten Verhaltens des Partners antizipiert und umgekehrt auf die Um-zu-Motive des Partners als echte Weil-Motive seines je eigenen Verhaltens hinzusehen fähig ist“ (Schütz 2004a: 312). Wendet man die Differenzierung von „auferlegten“ Weil- und „freien“ Um-zu-Motiven auf diese Aussage an, so ergibt sich zwangsläufig der eigentümlich anmutende Befund, dass in einer "partnerschaftlichen“ Beziehung, sämtliche Motive, die dem einen Partner „wesentlich“ sind, dem anderen „auferlegt" werden, et vice versa. ${ }^{14}$ Schütz illustriert seine Aussage anhand einer simp-

13 Im Gegensatz etwa zu einer philosophisch-erkenntniskritischen, wie man sie mit Bezug auf das vorliegende Problem auf der Basis von Kants Ausführungen zum „Dritten Widerstreit der transzendentalen Ideen“ (1993: B 472 ff.) betreiben könnte.

14 Schütz selbst zieht diese Schlussfolgerung in seinem Aufsatz „Der gut informierte Bürger“ (vgl. 2011a: 123). 
len Frage-Antwort-Relation: Der eine Partner einer Wirkensbeziehung stellt dem anderen eine Frage, um eine Antwort zu erhalten, und der andere antwortet, weil die Frage seines Partners durch das Ziel motiviert war, eine Antwort zu erhalten. Macht es aber, so möchte man Schütz fragen, in der Einschätzung der Beziehung nicht einen Unterschied, ob die beiden Partner sich auf einem wissenschaftlichen Kongress austauschen oder im Verhörzimmer?

Die beschriebene Problematik scheint Schütz und später auch Luckmann bewusst gewesen zu sein. Zumindest kann man deren oben konstatierten Widerwillen, sich bezüglich konkreter Beispiele in der Frage von Freiwilligkeit und Zwang eindeutig festzulegen, in dieser Richtung interpretieren. Betont wird stattdessen das Je-nachdem bzw. das Sowohl-als-auch diesbezüglicher Einschätzungen und hierfür wird ein Grund angeführt, der bereits in einem früheren Zitat von Luckmann anklang: „Jeder Erfahrungsablauf in seinen verschiedenen Dimensionen und jede Situation in ihren verschiedenen Aspekten weisen ein Ineinandergreifen und ein Zusammenspiel von auferlegten und motivierten Momenten auf. Dies gilt ... für die Relevanzstrukturen überhaupt“" (Schütz/Luckmann 2003: 265).

Diese Äußerung verweist auf die „Tatsache, daß sowohl thematische als auch interpretative Relevanz unlöslich mit Motivationsrelevanz verknüpft ist“ (ebd.: 286), und damit auf die übrigen Relevanztypen. Auch bezüglich dieser erscheint die theoretische Festlegung "freier" und "auferlegter" Relevanzen dem Alltagsverstand nicht immer als einleuchtend. Im Falle der thematischen Relevanz differenziert Schütz zwischen einer Reihe von Umständen, aufgrund derer sie als „auferlegt“ einzustufen sei. Als wichtigsten Fall kennzeichnet er denjenigen der „unvertraute[n] Erfahrung, die sich uns gerade wegen ihrer Unvertrautheit aufdrängt. Diese Erfahrung machen wir uns nicht durch einen Willensakt thematisch, und darum nennen wir diese Art der Relevanz ,auferlegte Relevanzen “ (Schütz 2004b: 91). Aber gibt es nicht Menschen, die sich willentlich in ihnen unvertraute Situationen begeben, um in respektive aus ihnen zu lernen (man denke z. B. an den ethnographischen Forscher)? Ist diesen die Zuwendung zum neuen Thema ,auferlegt“? Von freiwilliger Aufmerksamkeit spricht Schütz hingegen, wenn das Subjekt den Horizont eines sich bereits im "Griff des Bewusstseins“ befindlichen Themas ausdeutet, wenn es mit anderen Worten „die dem ausgezeichneten Thema wesentlich zugehörenden, impliziten thematischen Relevanzen explizit“ (ebd.: 95) macht, was er auch als „freiwillige Umwandlung von im Horizont verbliebenen in thematische Gegebenheiten" (ebd.: 96) bezeichnet. Doch erkennt Schütz selbst, wie oben bemerkt, dass die Vertiefung eines Themas durch dessen faktische Struktur weitgehend vorgegeben ist. Kann man angesichts dessen von einer thematischen Zuwendung aus freiem Willen sprechen? 
Für den Fall der Interpretationsrelevanz formuliert Luckmann:

„Bei routinemäßiger Deckung ist ,Interpretation' automatisch. Es erfolgt keine explizit urteilende Auslegung, in der einerseits das Thema, andererseits relevante Wissenselemente gesondert in den Griff des Bewußtseins kommen und miteinander ,verglichen werden. Diese Form der Interpretationsrelevanz gehört zur Kategorie der , auferlegten Relevanzen. Falls jedoch das Thema zum Problem wird, besteht ein Motiv zur mehr oder minder expliziten, schrittweisen, ,urteilenden' Auslegung. In diesem Fall handelt es sich um ,motivierte` Interpretationsrelevanz" (Schütz/Luckmann 2003: 273).

Auch an diesem Punkt erscheint die relevanztheoretische Redeweise kontraintuitiv. Gerade diejenigen auslegungsrelevanten Aspekte des Themas und des zuhandenen Wissensvorrats, die in einer als problematisch empfundenen Situation auftauchen, gelten als „frei“, während die unproblematische Auslegung als "gebunden“ bezeichnet wird. Erscheint mir nicht umgekehrt dasjenige, was sich meinen Deutungsroutinen fügt, als willfährig, und dasjenige, was sich ihnen widersetzt, als auferlegt? Wenn ein Individuum in einer ihm alltäglich vertrauten Situation spontan den Blick auf die bedeutsamen Aspekte des Themas richtet und die richtigen Wissenselemente zur Interpretation heranzieht, würden wir dann sagen, es bestimmt diese Relevanzen unfreiwillig? Und im gegenteiligen Fall, es bestimmt sie freiwillig?

\section{Theoretische Reflexion}

In der Hoffnung, den fraglichen Punkt klar gemacht zu haben, breche ich die Diskussion von Unverträglichkeiten, die sich bei der Kontrastierung von Alltagsverständnis und relevanztheoretischer Bestimmung von „auferlegten“ und „wesentlichen" Relevanzen ergeben, an dieser Stelle ab. Die Behauptung, für die das Vorige Evidenzen beizubringen versuchte, lautet, dass die betreffende phänomenologische Beschreibung subjektiver Bewusstseinsaktivitäten, innerhalb derer sich Wertzuschreibungen konstituieren, nur schwer vereinbar ist mit den Konstruktionen des Common Sense, derer wir uns im Alltag bedienen. So betrachtet verstößt die Sprachregelung der Relevanztheorie gegen das von Schütz formulierte Adäquanzpostulat, wonach „,jeder Ausdruck, der in einem wissenschaftlichen System benutzt wird, das sich auf menschliches Handeln bezieht, ... so konstruiert werden [muß], daß eine innerhalb der Lebenswelt durch einen individuell Handelnden geleistete Handlung, und zwar so, wie es die typische Konstruktion 
anzeigt, sowohl für den Handelnden selbst als auch für seine Mitmenschen vernünftig und verstehbar ist" (Schütz 2010: 226). Wie die obigen Beispiele zeigen, kann von einer solchen Verstehbarkeit im Falle der relevanztheoretischen Definition „auferlegter“ und „freier“ Relevanzen nicht ausgegangen werden. Indessen setzt die Anwendung des Adäquanzpostulats auf die Relevanztheorie voraus, dass es sich bei dieser um eine Konstruktion zweiter Ordnung handelt, sprich um eine wissenschaftliche Rekonstruktion vorwissenschaftlicher Sinnzusammenhänge, denn nur auf solche Theoreme bezieht sich das Postulat. Ob diese Voraussetzung erfüllt ist, ist jedoch auf den ersten Blick keineswegs unstrittig.

So kann man sich auf den Standpunkt stellen, bei phänomenologischer Deskription und soziologischer Rekonstruktion handele es sich um zwei grundverschiedene Dinge, die strikt voneinander zu trennen seien. Thomas Luckmann vertritt diese Sichtweise, wenn er die egologisch-reflexive Methode der Phänomenologie der kosmologisch-induktiven Vorgehensweise der Sozialwissenschaften gegenüberstellt und beide als „Parallelaktion“ bezeichnet (vgl. Luckmann 1979: 196; 2007a: 97; 2007b: 131). Doch darf über diese Grenzziehung nicht der eingangs dargestellte Umstand vergessen werden, dass die Lebenswelttheorie von Schütz gerade als philosophische Fundierung empirischer Begriffsbildungen entwickelt wurde, woraus folgt, dass sich sein Relevanzkonzept - wie auch dasjenige des Sinns oder der Handlung - durchaus im Hinblick auf seine Anwendbarkeit innerhalb empirischer Forschung hinterfragen lassen muss. Diese Feststellung stellt die prinzipielle Unterschiedlichkeit der Betrachtungsweisen nicht in Abrede, doch insistiert sie darauf, dass die phänomenologische Aufklärung von Bewusstseinsakten von Schütz letztlich im Hinblick auf soziologische Wirklichkeitsanalysen betrieben wurde und dass dieses Motiv bei ihrer Beurteilung nicht außen vor gelassen werden darf. ${ }^{15}$ Schütz philosophische Exkursionen weisen in diesem Sinne über sich selbst hinaus. ${ }^{16}$

Schütz selbst erkennt einen doppelten Lösungsansatz für das Relevanzproblem. Am Ende des „Sinnhaften Aufbaus“ verweist er auf das „Problem der Relevanz, dessen endgültige Klärung zwar nur auf dem Boden der allgemeinen phänomenologischen Analyse möglich sein wird, das aber dennoch vorerst auf sozialwissenschaftlichem Gebiet in Angriff genommen werden kann" (Schütz 2004a: 439). In

15 In die gleiche Richtung argumentiert Ilja Srubar in seiner Interpretation des Sinnhaften Aufbaus (1988: Kap. III).

16 Von daher erklärt sich Schütz' Übertragung der Husserl'schen Transzendental- in eine Mundanphänomenologie, die als Prolegomena einer Wirklichkeitswissenschaft auf dem Boden bestimmter Prämissen argumentiert, welche aus transzendentalphilosophischer Perspektive problematisch erscheinen mögen (wie z. B. die Existenz von Mitmenschen; vgl. Schütz 2009). 
seiner späteren, oben dargelegten Relevanztheorie versucht er sich an eben jener letztgültigen phänomenologischen Analyse, wobei im Hintergrund immer das Interesse an empirisch-sozialwissenschaftlichen Fragen steht. Zwar betreibt Schütz eine Beschreibung allgemeiner Bewusstseinsstrukturen, doch besteht darin nicht, wie bei Husserl, der eigentliche Zweck des Unterfangens. Vielmehr soll die Aufklärung der formalen Strukturen die empirische Analyse sozialer Akteure und ihres Handelns vorbereiten. Während Husserl mittels der phänomenologischen Reduktion auf das Allgemeine zielt, will Schütz philosophische Erkenntnisse für die Rekonstruktion der Sinnsetzungen konkreten Handelns nutzen. Man darf daher konstatieren, dass sich die Relevanztheorie von Schütz aufgrund ihres spezifischen Erkenntnisinteresses dem Adäquanzpostulat nicht verweigern kann. Obgleich es sich bei ihr nicht um eine Konstruktion zweiter Ordnung im eigentlichen Sinne handelt, sind ihr die Unverträglichkeiten mit dem Common Sense doch als Mangel anzulasten.

Worin liegt nun die Ursache dieses Defizits? Die These, die ich im Folgenden entwickeln möchte, besagt, dass der Grund in der phänomenologischen Methode als solcher zu suchen ist, oder genauer: in der Einnahme einer strikt egologischen Perspektive. Beginnen wir mit dem generellen Kriterium für die Einstufung einer Relevanz als „gebunden“ oder „frei“. Als solches gilt Schütz offenkundig die Disponibilität. Eine Relevanz ist demnach „auferlegt“, wenn der Handelnde sie im Moment des Handelns nicht beliebig verändern kann, und umgekehrt „wesentlich“, sofern er dies kann. Von dieser Überlegung her leuchtet z. B. die Definition von in der Vergangenheit angelegten Weil-Motiven als "auferlegt" und von in die Zukunft gerichteten Um-zu-Motiven als „frei“ unmittelbar ein. Auch wird deutlich, weshalb bereits früher getroffene Handlungsentwürfe als „gebunden" konzipiert werden. Und doch bleibt die Festlegung in ihrer Allgemeinheit fragwürdig: Bietet die Ausrichtung auf die Zukunft allein eine hinreichende Begründung dafür, Um-zu-Motive generell als „frei“ zu bestimmen?

Betrachtet man die Relevanztheorie im Gesamten, so fällt auf, dass das Adjektiv "auferlegt" praktisch durchgehend solchen Relevanzen zugedacht wird, die dem Subjekt nicht ins aktuelle Bewusstsein dringen. Paradigmatisch illustriert dies die Diskussion der Motivationsrelevanzen. Schütz erkennt keinerlei „Evidenz dafür, daß der Handelnde (Sinnsetzende) die Weil-Motive seines Handelns (Sinnsetzungsakte) überhaupt im Blick habe“ (ebd.: 266). In analoger Absicht konstatiert Luckmann, Einstellungen seien „meist schwer thematisierbar und dem reflektiven Bewußtsein nur schwer zugänglich" (Schütz/Luckmann 2003: 300). Formulierungen wie diese legen die Vermutung nahe, dass Schütz und Luckmann Handlungsdispositionen als „,auferlegt“ gelten, sofern sie latent bleiben, während umgekehrt 
nicht-latente Relevanzen als „frei“ definiert werden. Dies zeigt sich wiederum besonders deutlich im Falle der Motivationsrelevanzen, denn die „freien“ Umzu-Motive sind solche, auf die der sein Handeln Planende seine Aufmerksamkeit richtet. Diese Lesart lässt sich auf die sonstigen Relevanztypen übertragen.

Mit der Unterscheidung von Bewusstseinslatenz und -aktualität, d.h. „Zwischen dem ,In-den-Relevanzen-Leben' und dem ,Auf-die-Relevanzen-Hinsehen “" (ebd.), ist ein zweites Kriterium für die Einstufung des Freiheitscharakters von Relevanzen eingeführt: „Auferlegte“ Relevanzen sind nicht frei verfügbar, wenn sie sich nicht im „Griff des Bewusstseins“ befinden; umgekehrt sind Relevanzen „frei“, wenn sie aus der Latenz gehoben werden. Auch dieser Gedanke erscheint auf den ersten Blick nachvollziehbar, liegt doch in der Bewusstmachung einer Relevanz durch das handelnde Subjekt tatsächlich eine notwendige Prämisse für die willentliche „Manipulierung“ derselben. Und wiederum taucht die Frage auf, ob das Kriterium alleine als ausreichend betrachtet werden kann: Ist eine Relevanz, die dem Subjekt ins Bewusstsein dringt, alleine deswegen schon disponibel?

Die Antwort auf diese - ebenso wie auf die zuvor gestellte - Frage muss negativ ausfallen. Die Begründung der Auferlegtheit durch den Verweis auf Zeit und Latenz ist nicht hinreichend, denn sie kommt einer unzulässigen Engführung des Konzepts der Disponibilität gleich. Die der Schütz'schen Relevanztheorie innewohnende Tendenz, „gebundene“ Relevanzen mit in der Vergangenheit liegenden respektive mit latenten sowie „freie“ Relevanzen mit zukunftsorientierten respektive mit bewussten zu identifizieren, übersieht einen wesentlichen Bestimmungsfaktor, nämlich aus der menschlichen Interaktion erwachsenden, sprich sozialen Zwang. Will man darüber urteilen, ob die Relevanzsetzung eines Individuums aus freien Stücken geschieht oder nicht, genügt es nicht zu wissen, ob das Subjekt auf die fragliche Relevanz hinsieht oder diese in die Zukunft projiziert. Man muss zudem berücksichtigen, innerhalb welcher sozialen Konstellation die Setzung geschieht. So man vor philosophisch belasteten Begrifflichkeiten nicht zurückschreckt, mag man Ersteres als die „innere Freiheit“, Letzteres als die „äußere Freiheit" des Individuums bezeichnen.

Nun wird in der Relevanztheorie das Moment des sozialen Zwanges ausdrücklich erwähnt, allerdings - und damit komme ich auf die oben angekündigte Diskrepanz zwischen dem Schütz'schen Manuskript und dessen Ausarbeitung durch Luckmann zu sprechen - ausschließlich in den "Strukturen der Lebenswelt“. ${ }^{17}$ Werfen wir einen Blick auf Luckmanns diesbezügliche Aussagen:

17 Ausgenommen eine einzige Stelle in Schütz' Manuskript (2004b: 83) im Kontext der Diskussion des Problems des Carneades. 
"Zuletzt ist noch eine weitere Form der , auferlegten' thematischen Relevanz zu erwähnen, nämlich die soziale , auferlegte'. Sie ist im täglichen Leben von der größten Bedeutung. Das Handeln der Mitmenschen - und zwar sowohl die Handlungsabläufe wie die Handlungsresultate - setzen dem einzelnen Themen vor, denen er sich zuwenden muß. Diese Form der, auferlegten' thematischen Relevanz überschneidet sich allerdings mit den anderen. Handlungsabläufe und Handlungsresultate können zum Beispiel völlig unerwartet sein; sie fügen sich nicht in die routinierten Erwartungen, die auf Mitmenschen bezogen sind, ein. In diesem Fall handelt es sich offensichtlich um das Hervorstechen bzw. Abgehobensein von Unvertrautem gegenüber einem Hintergrund von Vertrautem. Aber Mitmenschen können auch in einen Erfahrungsablauf eingreifen, dem einzelnen neue Themen aufzwingen, ohne daß ihr Handeln in nachfolgender Interpretation als atypisch, unvertraut erscheint“ (Schütz/Luckmann 2003: 262).

Tatsächlich ist dies die einzige Passage in den relevanztheoretischen Erörterungen von Luckmann oder Schütz, in der einigermaßen ausführlich auf sozial aufgezwungene Relevanzen eingegangen wird, und dies auch nur im Hinblick auf den Typus der thematischen Relevanz. Dabei wird der sozial „auferlegten“ thematischen Relevanz kein eigener systematischer Ort innerhalb der Theoriearchitektur zugewiesen, vielmehr wird ihre Überschneidung mit den übrigen, nicht-sozialen hervorgehoben. Es ist in diesem Kontext bezeichnend, dass eine erste Unterart der sozial „auferlegten“ thematischen Relevanz mit Fällen in Zusammenhang gebracht wird, in denen sich Mitmenschen in einer Art und Weise verhalten, die den Erwartungen des Relevanzsetzenden widerspricht, d.h. der Zwang wird hier überführt in das Problem von Vertrautheit und Unvertrautheit. Fälle hingegen, in denen Menschen ihre gesellschaftliche Macht dazu nutzen, anderen die Beschäftigung mit einem Thema aufzuzwingen, werden in einem einzigen, in eigentümlicher Weise verklausulierten Satz abgehandelt.

Auch wenn man also unterstellt, dass Luckmann mit der zitierten Passage auf die Unvollendetheit des Schütz'schen Relevanzmanuskripts reagierte, so muss man doch festhalten, dass seine Erörterung für eine angemessene Darstellung des Aspekts der „äußeren Freiheit“ bei weitem nicht ausreicht. Generell ist zu konstatieren, dass die Relevanztheorie in ihrer vorliegenden Form das Problem der sozialen Auferlegtheit von Relevanzen nur unzureichend berücksichtigt. Die Ursache scheint klar und wird von Schütz selbst benannt: Es ist die methodologisch gewollte Fokussierung auf das einzelne Individuum, welches „die Welt der Natur ohne Verbindung mit seinen Nebenmenschen" (Schütz 2004b: 130) erfährt; eine Vorgehensweise, die „um der vereinfachten Darstellung ... willen“ (ebd.) geschieht. Dem entspricht die Illustrierung der Problematik mittels des Beispiels 
des Carneades (vgl. ebd.: 80 ff.; Schütz/Luckmann 2003: 252 ff.), in dem ein einzelner Mann die Objektwelt des Zimmers, in dem er sich befindet, ausdeutet das bedeutet anhand eines Falls, in dem soziale Beziehungen allenfalls am Rande eine Rolle spielen. Schütz beginnt seine Ausführungen bewusst mit der „Fiktion, daß dieses Problem [der Relevanz; A. G.] für ein als isoliert angenommenes Bewußtsein ohne irgendeinen Bezug auf Sozialität untersucht werden kann" (Schütz 2004b: 180). Analog zum Vorgehen im „Sinnhaften Aufbau“ beabsichtigte er, in späteren Kapiteln den Untersuchungskreis auf die soziale Sphäre auszuweiten, und er war sich dessen bewusst, dass die „Relevanzbegriffe und ihre Verknüpfungen ... vollständig revidiert werden müssen, sobald der Begriff der Intersubjektivität eingeführt worden ist. Die als selbstverständlich hingenommene Welt ist nicht meine private Welt, und die Relevanzsysteme sind zum Großteil nicht meine private Angelegenheit" (ebd.: 130). Doch hat er die betreffenden Teile nie geschrieben.

Von daher liegt die Annahme nahe, man könne den diagnostizierten Mangel der Relevanztheorie einfach durch die von Schütz' intendierte, jedoch nicht mehr vollzogene Ausweitung des Betrachtungshorizonts auf die soziale Sphäre beheben. Derart würde das Moment der „äußeren Freiheit“ thematisierbar und somit die Engführung des Disponibilitätskonzepts revidierbar. Die entsprechend erweiterte Sprachregelung läge näher am Common Sense und garantierte die vom Adäquanzpostulat eingeforderte Übersetzbarkeit in Konstruktionen erster Ordnung, wohingegen die Gleichsetzung von in der Vergangenheit angelegten respektive von latenten mit „auferlegten“ Motiven im Hinblick auf die sozialwissenschaftliche Rekonstruktion von Alltagskonstruktionen bestenfalls unergiebig und schlimmstenfalls irreführend ist.

Meine Behauptung lautet nun, dass es zusätzlich zu einer solchen Erweiterung des Bruchs mit der rein egologischen Perspektive bedarf, um das Moment des sozialen Zwanges in der Relevanztheorie angemessen reflektieren zu können. Dabei folge ich jenem thematischen Verweis, der in der Rede von „auferlegten“ Relevanzen implizit enthalten ist, nämlich dem Verweis auf das Phänomen der Macht, und ich rekurriere auf dasjenige Theoriegebäude, dessen Fundament Schütz mittels seiner phänomenologischen Reflexionen errichten wollte: auf die Soziologie von Max Weber. Für diesen bedeutet Macht ,jede Chance, innerhalb einer sozialen Beziehung den eignen Willen auch gegen Widerstreben durchzusetzen, gleichviel worauf diese Chance beruht" (Weber 1980: 28). An dieser Definition ist im vorliegenden Zusammenhang die Formulierung "auch gegen Widerstreben" von Interesse, denn aus ihr ist im Umkehrschluss abzuleiten, dass in soziologischer Absicht von Macht auch dann zu sprechen ist, wenn derjenige, über 
den Macht ausgeübt wird, keinen dem Machtstreben zuwiderlaufenden Willen erkennen lässt. Relevanztheoretisch formuliert bedeutet dies, dass Macht nicht bloß auf die Art ausgeübt werden kann, dass ein Akteur einem anderen die eigenen Relevanzen wider dessen Willen aufzwingt, sondern ebenso in der Weise, dass ein Akteur die eigenen Relevanzen als gemeinsame definiert und als solche darzustellen weiß.

Aus dieser Erkenntnis ergibt sich für die egologische Betrachtung ein grundlegendes Problem: Wenn Machtbeziehungen unabhängig davon bestehen, ob der „Unterworfene“ in sie einwilligt oder nicht, wie kann man sie dann anhand der bloßen Beschreibung von dessen Bewusstseinsprozessen analysieren? Gewährt der von Schütz für seine Relevanztheorie gewählte Ansatz prinzipiell eine hinreichende Basis für eine Aufklärung von Machtbeziehungen, d. h. hier im Hinblick auf den Zwangs- bzw. Freiwilligkeitscharakter von Relevanzen? Oder um es anhand eines bekannten Beispiels auszudrücken: Genügt die phänomenologische Deskription der Bewusstseinsinhalte des Weber'schen Holzhackers, um beurteilen zu können, ob dieser „gegen Lohn oder aber für seinen Eigenbedarf oder zu seiner Erholung" (ebd.: 4) Holz hackt und ob ihm die je entsprechenden Relevanzen „auferlegt" oder „wesentlich“ sind? Mir scheint, dass ein Ja auf alle diese Fragen nur unter der Annahme möglich ist, dass das Subjekt von den „objektiven“ gesellschaftlichen (Macht-)Strukturen, innerhalb derer es steht, keine "falschen“ Vorstellungen haben kann - eine Prämisse, die äußerst fragwürdig ist.

Anstatt entsprechende empirische Gegenbeispiele zu entwerfen, konzentriere ich mich auf eine theoretische Widerlegung. Akzeptiert man Schütz' These, wonach das Subjekt vornehmlich die „freien“ Um-zu-Motive im „Griff seines Bewusstseins“ hat, während ihm die „auferlegten“ Weil-Motive meist verborgen bleiben, dann muss man konsequenterweise folgern, dass der Mensch mit einer systematischen Überschätzung seiner Willens- respektive Handlungsfreiheit durch die (Lebens-)Welt geht. Sollte man als wissenschaftlicher Beobachter der Wahrnehmung eines solchen Subjekts vertrauen? Und wie will man, sich die Weber'sche Machtdefinition in Erinnerung rufend, im Zuge eines rein introspektiven Verfahrens unterscheiden, ob derjenige, der gemäß Relevanzen handelt, die er für genuin eigene hält, nicht in Wirklichkeit einer Form struktureller Gewalt unterliegt, um die er nicht weiß? Das setzte voraus, dass man im Zuge phänomenologischer Reduktion fremde Interessen von eigenen trennen kann, dass man - in Analogie zum kantischen Ich denke, das alle meine Vorstellungen begleiten können muss (vgl. Kant 1993: B 131) - innerhalb der primordialen Sphäre des Subjekts ein Ich will freilegen kann, das alle meine Intentionen begleitet und allen auferlegten Intentionen fehlt. 
Es scheint mir kein Zufall zu sein, dass man im Werk Edmund Husserls, auf dessen Bewusstseinsanalysen sich Schütz bezieht, keine genuin phänomenologischen Konzeptionalisierungen von Zwang oder Macht findet. Man kann diesen Umstand dahingehend interpretieren, dass Husserl erkannt hat, dass die Aufklärung des Machtphänomens prinzipiell außerhalb der Reichweite introspektiver Verfahren liegt. Macht ist ein Relationsbegriff, und das in ihm ausgedrückte Phänomen ist durch die alleinige Betrachtung der einen Seite der Relation, soll heißen mittels egologischer Reflexion, nicht angemessen zu erörtern. Vielmehr läuft man auf diesem Wege Gefahr, die oben angedeutete „Selbsttäuschung“ des handelnden Subjekts zu objektivieren. ${ }^{18}$

Bezüglich Letzterer war im Obigen in unangemessen laxer Art und Weise von "falschen“ Vorstellungen des Subjekts über seine soziale Situierung die Rede. Die Präzisierung dieser Redeweise bietet die Gelegenheit, Schütz' eigene Position zum Verhältnis von egologischer und soziologischer Perspektive in die Diskussion einzubeziehen. In seiner Terminologie, die er - mit abgewandeltem Inhalt - von Weber übernimmt, lässt sich das Problem mittels der Unterscheidung von subjektivem und objektivem Sinn fassen. ${ }^{19}$ Der subjektive Sinn ist derjenige Sinn, den der Handelnde selbst mit seinem Tun verbindet, der objektive Sinn hingegen derjenige, den ein Beobachter desselben Handelns erkennt. Beide sind nach Schütz schlechthin nicht ineinander überführbar, weil ihre Erkenntnis grundlegend unterschiedliche Arten der Zuwendung erfordert:

„Vom subjektiven Sinn eines Erzeugnisses sprechen wir dann, wenn wir den Sinnzusammenhang im Blick haben, in welchem die Erlebnisse des Erzeugenden, von denen das Erzeugnis zeugt, für diesen stehen oder standen, d. h. wenn wir die polythetischen Akte, in denen sich diese Erlebnisse des das Erzeugnis Setzenden aufbauten, in Gleichzeitigkeit oder Quasigleichzeitigkeit unserer Dauer nachzuvollziehen vermögen. ... Objektiven Sinn können wir hingegen nur einem Erzeugnis als solchem prädizieren, also dem fertig konstituierten Sinnzusammenhang des Erzeugten selbst, dessen Erzeugung in polythetisch aufbauenden Akten im fremden Bewußtseins von uns unbeachtet bleibt“" (Schütz 2004a: 269).

18 Schütz' Aussage, wonach „auferlegte Relevanzen ein abgeleiteter Typus wesentlicher Relevanzen, sozusagen Relevanzen zweiter Ordnung" (Schütz 2004b: 123) sind, kann als Tendenz in diese Richtung gelesen werden.

19 Damit folge ich dem Verweis auf den „Sinnhaften Aufbau“, den Elisabeth List, die Herausgeberin des Relevanzmanuskripts im Rahmen der ASW, im Hinblick auf dessen mögliche „Vollendung“ gibt. 
Übertragen wir diese Überlegung auf das in Frage stehende Problem. Eine soziale Beziehung, innerhalb derer sich ein Subjekt befindet, hat für dieses selbst einen Sinn, der prinzipiell von demjenigen Sinn abweicht, den ein Außenstehender in dieser Beziehung erkennt. Das umfasst auch die Frage der Relevanzen. Hinsichtlich der Frage, welche Bestandteile des in der Beziehung geteilten Relevanzsystems welchem Partner auferlegt sind, wird der Beteiligte selbst zu einem ganz anderen Urteil kommen als der Beobachter. ${ }^{20}$ Da für Schütz weiterhin klar ist, dass „die Welt des objektiven Sinnes ... auch in der sozialen Sphäre losgelöst [ist] von den Konstitutionsprozessen eines sinngebenden ... Bewußtseins“ (ebd.: 122), folgt, dass die phänomenologische Beschreibung des subjektiven Bewusstseins zur Analyse „objektiver“ Machtverhältnisse nicht hinreichen kann. Die prinzipielle Grenze zwischen subjektivem und objektivem Sinn zeigt sich im Besonderen bezüglich der Rekonstruktion von Motivationsrelevanzen, bei der vorliegender Aufsatz seinen Ausgang nahm: „Das Aufsuchen der ,Motive“ erfolgt von dem dem Beobachter gegebenen objektiven Sinne her so, als ob dieser objektive Sinn, der vom Handelnden (und zwar von diesem fraglos) gemeinte Sinn wäre. ... Daneben aber bleibt es unzweifelhaft, daß sich eben dieser ,gemeinte' Sinn im motivationsmäßigen Verstehen ebensowenig wie im aktuellen Verstehen erschließt. Beiden Arten des Verstehens ist Sinn nur als objektiver Sinn zur Deutung vorgegeben“" (ebd.: 113). Das bedeutet im Umkehrschluss, dass „eine Verifikation meiner Interpretation des fremden Erlebens an der Selbstinterpretation des Beobachteten unvollziehbar" (ebd.: 327) ist, und in der Folge, dass hinsichtlich einer die Auferlegtheit von Relevanzen betreffenden Einschätzung nicht ausschließlich auf die Sichtweise des Subjekts gebaut werden kann. ${ }^{21}$

Im Hinblick auf die Frage, inwieweit die phänomenologische Konstitutionsanalyse bei der Rekonstruktion sozialer Machtbeziehungen behilflich sein kann, ist demzufolge Zurückhaltung angeraten. Die egologisch vorgehende phänomenologische Deskription, die alter als bloßen Bewusstseinsinhalt von ego konzipiert, greift konzeptionell zu kurz, um das Phänomen der Macht, das in seinem Kern ein relationales, intersubjektives ist, adäquat behandeln zu können. Den „,isolierten Erfahrungen des Individuums“ (Schütz 2004b: 112) ist es nicht anzusehen, ob seine Relevanzsetzungen in einem Kontext sozialen Zwangs oder gesellschaftlicher Freiheit geschehen. Gleichwohl bietet die phänomenologische

20 Vgl. hierzu Schütz' Gleichheits-Aufsatz, wo das analoge Problem auf der Ebene der Beziehung zwischen Eigen- und Fremdgruppe behandelt wird (Schütz 2011b).

21 Von daher ist es angebracht, wenn Schütz selbst im Zuge einer Auflistung der Leistungen der Relevanztheorie die Aufklärung von Machtphänomenen auslässt (vgl. Schütz 2004b: 128). 
Aufklärung des Relevanzproblems der sozialwissenschaftlichen Forschung ein wertvolles Instrumentarium. Das betrifft erstens ihren heuristischen Wert. Die formale Analyse legt die allgemeine Struktur von Relevanzsetzungen offen und weist damit dem Wirklichkeitswissenschaftler den Weg, den er bei der Rekonstruktion empirischer Relevanzsysteme zu gehen hat. Die Relevanztheorie stellt der empirischen Forschung mit anderen Worten eine Art „Erkennungsraster" zur Verfügung, der allgemein anwendbar ist. Aus dieser Offenlegung einer „mathesis universalis“ erwächst der zweite forschungspraktische Vorzug, denn der Rekurs auf ein generelles Muster, das sämtliche sozio-historischen Konstruktionen teilen, ermöglicht erst deren systematische Kontrastierung.

Aus theoretischer Sicht beugt die phänomenologische Aufklärung zudem einer irreführenden, reifizierenden Sichtweise sozialer Relevanzsysteme entgegen, welche diesen den Charakter einer Realität sui generis zugesteht (vgl. Durkheim 2007). Demgegenüber ist auf der Einsicht Schütz' zu bestehen, dass sozio-kulturell verfestigte Relevanzstrukturen in ihrer Genese stets auf die konstitutiven Akte von Individuen verweisen, die daher die Aufmerksamkeit der Soziologie verdienen: „Die maximale Verdeutlichung und Explizierung dessen, was gemeinhin von den in der Sozialwelt Lebenden über diese gedacht wird, ist ... erstes Ziel jeder Sozialwissenschaft und somit auch der verstehenden Soziologie" (Schütz 2004a: 405). Das Verstehen subjektiver Sinnvollzüge ist Voraussetzung für die sinnadäquate Erklärung sozialer Phänomene. Hinsichtlich der vorliegenden Problematik bedeutet dies, dass eine soziologische Machtkonzeption, welche ausschließlich objektive Strukturen berücksichtigt und im Gegenzug deren subjektive Bedeutung außer Acht lässt, der sozialen Realität nicht gerecht wird (vgl. Dreher 2012).

Umgekehrt reicht der subjektivistische Ansatz alleine nicht hin. Ausschließlich im Spannungsfeld von Konstitution und Konstruktion sind gesellschaftliche Relevanzsysteme adäquat zu untersuchen, gerade auch im Hinblick auf den Aspekt der Macht. Die von Berger und Luckmann betonte dialektische Beziehung zwischen Individuum und Gesellschaft (vgl. Berger/Luckmann 1998: 65) darf nicht einseitig aufgelöst werden - weder hin zur bloßen Betrachtung der Seite der Gesellschaft, die den Sinn von Institutionen rein funktionalistisch in diesen selbst verortet, noch hin zur bloßen Betrachtung der Seite des Individuums, wo lediglich der subjektiv gemeinte Sinn sozialer Beziehungen zu finden ist. Stattdessen gilt es, Anknüpfungspunkte zwischen beiden Sichtweisen zu suchen, wie sie mit Bezug auf das hier untersuchte Problem etwa in Schütz' Beschreibung der Wissenssedimentierung und des dabei wirksamen Moments der Autorität zu finden sind. Auf diese Art mag die Schütz'sche Relevanztheorie als Ansatzpunkt dienen 
für die nach wie vor ausstehende phänomenologisch fundierte Analytik sozialer Machtstrukturen (vgl. Endreß 2008).

\section{Fazit}

Die vorangegangenen Ausführungen galten einem kritischen Weiter-Denken der Schütz'schen Relevanztheorie im Hinblick auf eine soziologische Handlungsund Machtanalyse. Sie nahmen ihren Ausgang an der Gegenüberstellung eines von Schütz gewählten Beispiels mit einer Szene aus der fiktionalen Sinnwelt des (Hollywood-)Films. Die nähere Betrachtung förderte eine ganze Reihe von Unverträglichkeiten zwischen der relevanztheoretischen und der alltagssprachlichen Verwendung der Adjektive „auferlegt“ und „frei“ zutage, als deren Ursache eine Engführung des Freiheitsbegriffs in der Theorie von Schütz erkannt wurde. Diese Engführung wiederum wurde als Folge des von Schütz gewählten, phänomenologischen Problemzugangs identifiziert. Letzterer, so die These, ist aufgrund seiner egologischen Methode prinzipiell nicht in der Lage, gesellschaftliche Machtstrukturen, wie sie untrennbar mit sozialen Relevanzsystemen verbunden sind, adäquat zu erfassen.

Die damit vorgeschlagene "machttheoretische“ Lesart der Relevanztheorie entspricht nun nicht Schütz' eigener Intention - seine Theorie wird hier sozusagen am fremden Leisten gemessen. Doch ergibt sie sich beinahe zwangsläufig, wenn man bestimmte Implikationen seines Sprachgebrauchs weiterverfolgt, d.h. eine Sub-Thematisierung im oben definierten Sinne vornimmt. Vergleichbares hat Schütz gegen Ende seines Lebens selbst getan, indem er das Konzept auferlegter Relevanzen für eine Analyse gesellschaftlicher Diskriminierung verwendete (vgl. Schütz 2011b), ohne allerdings die Übertragung phänomenologischer Begriffe auf Gruppenprozesse methodologisch zu klären.

Das Ergebnis der Überlegungen bestärkt somit Luckmanns Erkenntnis, dass es sich bei phänomenologischer Reflexion und soziologischer Rekonstruktion um prinzipiell gegenläufige Vorgehensweisen handelt: Erstere zielt unter Absehung von kontingenten Inhalten des Bewusstseins auf dessen formale Strukturen, Letztere interessiert sich umgekehrt gerade für die konkreten, kulturell geformten Inhalte dieser Strukturen. Von daher ist der in der Schütz'schen Theorie, wenn vielleicht nicht intendierte, so doch angelegte Versuch, gleichsam a priori zu bestimmen, welche Relevanzen als auferlegt und welche als frei zu gelten haben, aus soziologischer Sicht fehlgeleitet. Zum Zwecke der empirischen Beschreibung 
sozialer Wirklichkeit ist das terminologische Instrumentarium hinsichtlich der Machtfrage neutral zu halten.

Im Rückblick auf die Filmszene, bei welcher die Argumentation ihren Anfang nahm, lässt sich abschließend festhalten, dass der im Regen singende und tanzende Don Lockwood zweierlei illustriert. Er macht klar, dass verliebte Menschen sowohl ihrer Umwelt - wie etwa dem verdutzten Straßenpolizisten - als auch der Sozialtheorie zum Problem werden können. In ersterem Falle wird die Schütz'sche Relevanztheorie kaum eine Hilfe sein können, in letzterem hingegen vermag sie einem wertvolle Einsichten an die Hand zu geben, die jedoch - so die These des Vorangegangenen - ohne die Berücksichtigung der Ebene der Konstruktion zu Schlussfolgerungen führen können, die dem Common Sense höchst unplausibel erscheinen. Von daher gilt das Plädoyer einer Erweiterung der Schütz'schen Konzeption, die sich in Übereinstimmung mit dem „Postulat der Adäquanz“ am Leitfaden des Alltagsverstandes orientiert.

\section{Literatur}

Berger, Peter L. und Thomas Luckmann (1998), Die gesellschaftliche Konstruktion der Wirklichkeit. Eine Theorie der Wissenssoziologie, Frankfurt a. M.: Fischer

Dreher, Jochen (2012), Zur lebensweltlichen Konstitution sozialer Ungleichheit und symbolischer Machthierarchien, im vorliegenden Bd., S. 125-145

Durkheim, Emile (2007), Die Regeln der soziologischen Methode, Frankfurt a. M.: Suhrkamp

Endreß, Martin (2008), Reflexive Wissenssoziologie als Sozialtheorie und Gesellschaftsanalyse. Zur phänomenologisch fundierten Analytik von Vergesellschaftungsprozessen, in: Jürgen Raab et al. (Hg.), Phänomenologie und Soziologie. Theoretische Positionen, aktuelle Problemfelder und empirische Umsetzungen, Wiesbaden, S. 85-95

Kant, Immanuel (1993), Kritik der reinen Vernunft, Hamburg: Meiner

List, Elisabeth (2004), Einleitung der Herausgeberin, in: Alfred Schütz Werkausgabe, Bd. VI.1: Relevanz und Handeln 1. Zur Phänomenologie des Alltagswissens, Kon$\operatorname{stanz}$, S. 7-4O

Luckmann, Thomas (1979), Phänomenologie und Soziologie, in: Walter M. Sprondel/Richard Grathoff (Hg.), Alfred Schütz und die Idee des Alltags in den Sozialwissenschaften, Stuttgart, S. 196-206

Luckmann, Thomas (2007a), Aspekte einer Theorie der Sozialkommunikation, in: ders., Lebenswelt, Identität und Gesellschaft. Schriften zur Wissens- und Protosoziologie, Konstanz, S. 91-111

Luckmann, Thomas (2007b), Wirklichkeiten: individuelle Konstitution, gesellschaftliche Konstruktion, in: ders., Lebenswelt, Identität und Gesellschaft. Schriften zur Wissensund Protosoziologie, Konstanz, S. 127-137 
Schütz, Alfred (1971), Über die mannigfaltigen Wirklichkeiten, in: ders., Gesammelte Aufsätze, Bd. I: Das Problem der sozialen Wirklichkeit, Den Haag, S. 237-298

Schütz, Alfred (2003a), Symbol, Wirklichkeit und Gesellschaft, in: ders., Alfred Schütz Werkausgabe, Bd. V.2: Theorie der Lebenswelt. Die kommunikative Ordnung der Lebenswelt, Konstanz, S. 119-197

Schütz, Alfred (2003b), Über die manngifaltigen Wirklichkeiten, in: ders., Alfred Schütz Werkausgabe, Bd. V.1: Theorie der Lebenswelt 1. Die pragmatische Schichtung der Lebenswelt, Konstanz, S. 181-239

Schütz, Alfred (2004a), Der sinnhafte Aufbau der sozialen Welt. Eine Einleitung in die verstehende Soziologie (Alfred Schütz Werkausgabe, Bd. II), Konstanz: UVK

Schütz, Alfred (2004b), Das Problem der Relevanz, in: ders., Alfred Schütz Werkausgabe, Bd. VI.1: Relevanz und Handeln 1. Zur Phänomenologie des Alltagswissens, Konstanz, S. 65-228

Schütz, Alfred (2009), Das Problem der transzendentalen Intersubjektivität bei Husserl, in: ders., Alfred Schütz Werkausgabe, Bd. III.1: Philosophisch-phänomenologische Schriften 1. Zur Kritik der Phänomenologie Edmund Husserls, Konstanz, S. 227-256

Schütz, Alfred (2010), Das Problem der Rationalität in der Sozialwelt, in: ders., Alfred Schütz Werkausgabe, Bd. IV: Zur Methodologie der Sozialwissenschaften, Konstanz, S. 203-233

Schütz, Alfred (2011a), Der gut informierte Bürger. Ein Versuch über die soziale Verteilung des Wissens, in: ders., Alfred Schütz Werkausgabe, Bd. VI.2: Relevanz und Handeln 2. Gesellschaftliches Wissen und politisches Handeln, Konstanz, 115-129

Schütz, Alfred (2011b), Gleichheit und die Sinnstruktur der sozialen Welt, in: ders., Alfred Schütz Werkausgabe, Bd. VI.2: Relevanz und Handeln 2. Gesellschaftliches Wissen und politisches Handeln, Konstanz, S. 175-228

Schütz, Alfred und Thomas Luckmann (2003), Strukturen der Lebenswelt. Konstanz: UVK Srubar, Ilja (1988) Kosmion. Die Genese der pragmatischen Lebenswelttheorie von Alfred Schütz und ihr anthropologischer Hintergrund. Frankfurt a. M.: Suhrkamp.

Srubar, Ilja (2007), Wertbeziehung und Relevanz. Zu Alfred Schütz' Weber-Rezeption, in: ders., Phänomenologie und soziologische Theorie. Aufsätze zur pragmatischen Lebenswelttheorie, Wiesbaden, S. 151-171

Weber, Max (1980), Wirtschaft und Gesellschaft. Grundriß der verstehenden Soziologie. Tübingen: Mohr

Weber, Max (1988), Die Wirtschaftsethik der Weltreligionen, in: ders., Gesammelte Aufsätze zur Religionssoziologie, Bd. I, Tübingen, S. 237-573 